\title{
Evaluation of an Alternative Staining Method Using SYTO 13 to Determine Reticulated Platelets
}

\author{
Laura Hille$^{1}$ Marco Cederqvist ${ }^{1}$ Julia Hromek ${ }^{1}$ Christian Stratz ${ }^{1, *}$ Dietmar Trenk ${ }^{1}$ Thomas G. Nührenberg ${ }^{2}$
}

\footnotetext{
${ }^{1}$ Department of Cardiology and Angiology II, Clinical Pharmacology, University Heart Centre Freiburg • Bad Krozingen, Bad Krozingen, Germany

2 Department of Cardiology and Angiology II, University Heart Centre Freiburg • Bad Krozingen, Bad Krozingen, Germany

Thromb Haemost 2019;119:779-785.
}

\author{
Address for correspondence Thomas G. Nührenberg, MD, Department \\ of Cardiology and Angiology II, University Heart Centre Freiburg • Bad \\ Krozingen, Südring 15, D-79189 Bad Krozingen, Germany \\ (e-mail: Thomas.Nuehrenberg@universitaets-herzzentrum.de).
}

\section{Abstract}

\section{Keywords}

- reticulated platelets

- immature platelet fraction

- staining
Reticulated platelets reflect the rate of platelet turnover and represent the youngest circulating platelets in peripheral blood. Reticulated platelets contain residual ribonucleic acid (RNA) from megakaryocytes which is lost in a time-dependent manner and can be transcribed into proteins even in the absence of a nucleus. An increased proportion of reticulated platelets is associated with higher platelet reactivity, cardiovascular events and mortality. At present, a fully automated assay system (SYSMEX haematology analyser) is available for analysis. This method, however, is not suitable for extended laboratory investigations like subsequent cell sorting. Flow cytometry analysis after staining with thiazole orange (TO) is frequently used in such settings despite several limitations. Here, we describe a new assay for determination of reticulated platelets by flow cytometry using the nucleic acid staining dye SYTO 13 and compare it with SYSMEX and TO staining as current standards. A significant correlation between immature platelet fraction (IPF) determined by SYSMEX XE-2100 analyser and results obtained with the SYTO 13-based assay was observed $(r=0.668, p<0.001)$ which was stable during a reasonable time period. In contrast, the correlation between TO staining and IPF was weaker $(r=0.478, p=0.029)$ and lost after 90 minutes of staining. SYTO 13 staining of platelets enabled sorting of RNAlow and RNArich platelets which was confirmed by RNA quantification of sorted platelets. Except for fixation of platelets, sorting of these platelet sub-populations was stable under various experimental settings. In summary, determination of reticulated platelets with the new SYTO 13 assay offers distinct technical advantages enabling further laboratory processing.

\section{Introduction}

Reticulated platelets (RPs), also known as immature platelets, are a fraction of the platelet pool, mostly representing the youngest platelets released from the bone marrow. ${ }^{1,2}$ They are characterised by some-known so far-distinct features such as: increased ribonucleic acid (RNA) content, higher volume,

\footnotetext{
* Christian Stratz current address is Novartis AG, Fabrikstrasse, Basel, Switzerland.
}

received

November 1, 2018

accepted after revision

January 19, 2019

more dense granules, higher levels of surface activation markers and probably higher platelet reactivity. ${ }^{1,3-6}$ Until now, several studies have shown an association of RP levels and cardiovascular events or mortality. ${ }^{7-10}$ Several laboratory methods with distinct features are used to determine RP in clinical settings. Initially, analysis of RP was performed by means of light microscopy using supravital staining of blood with new methylene blue, but this method is obviously not well suited for extended applications. ${ }^{11}$ Currently, flow cytometry is the method preferred by the majority of clinical laboratories.

(C) 2019 Georg Thieme Verlag KG Stuttgart . New York
DOI https://doi.org/ 10.1055/s-0039-1681101. ISSN 0340-6245. 
Kienast and Schmitz reported a flow cytometric assay based on thiazole orange (TO) staining for analysing RP. ${ }^{12}$ TO binds to platelet RNA and various intracellular components. ${ }^{13}$ The amount of unspecific non-RNA binding and hence varying fluorescence intensity is affected by staining conditions, such as incubation time, temperature or fixation. ${ }^{1}$ Consequently, this method is hampered by an evident lack of analytical standardization, which disables a direct comparison of data obtained by different laboratories. ${ }^{4,12,14-16}$ Moreover, when prolonged sample work-up is inevitable, for example, in the setting of cell sorting with a low number of events, TO staining is difficult as it leads to unspecific TO-positive platelets and increasing fluorescence intensity over time.

An alternative option to measure RP is a fully automated assay using a fluorescent polymethine dye which has been established on the SYSMEX XE-2100 (SYSMEX, Kobe, Japan) analyser. The immature platelet fraction (IPF, \%) provides the fraction of immature platelets within the whole platelet pool, while immature platelet count provides the number of immature platelets $\left(10^{3} / \mu \mathrm{L}\right)$-both determined using a cutoff pre-defined by the manufacturer. By means of this method, an association between RP and clinical events or drug response, for example, in patients treated with thienopyridines, has previously been shown..$^{5,8-10,17}$ However, as the SYSMEX assay is a fully automated closed system, assay modifications for experimental purposes can hardly be implemented and more in-depth investigations on RP surface markers are not possible.

Therefore, the aim of our present work is to establish a new platelet staining protocol which overcomes some of the limitations in RP analysis mentioned before. A potential staining dye is SYTO 13 which is a cell-permeant green fluorescent dye with high potency and affinity to RNA showing a large fluorescence enhancement after binding. SYTO 13 has an absorption maximum at $491 \mathrm{~nm}$ and an emission maximum at $514 \mathrm{~nm}$ in the presence of RNA.

\section{Materials and Methods}

\section{Staining of Platelets}

We developed a laboratory protocol for SYTO 13 staining of platelets. First, washed platelets (WPs) were obtained from citrate-anticoagulated blood (Sarstedt, Nümbrecht, Germany). Blood was centrifuged at $150 \times \mathrm{g}$ for 10 minutes and plateletrich plasma (PRP) was manually separated. To avoid activation and aggregation of platelets, $10 \mu \mathrm{L}$ prostaglandin $\mathrm{I}_{2}(10 \mu \mathrm{g} / \mathrm{mL}$; Sigma-Aldrich, St. Louis, Missouri, United States) were added to $490 \mu \mathrm{L}$ PRP and thoroughly mixed. After pelleting $(380 \times \mathrm{g}$, 20 minutes), platelets were re-suspended in $1,000 \mu \mathrm{L}$ Tyrode'sHEPES buffer (pH 7.4; $145 \mathrm{mMol} \mathrm{NaCl}$ [Sigma-Aldrich], 2.9 mMol KCl [Sigma-Aldrich], 10 mMol HEPES [Sigma-Aldrich], 1

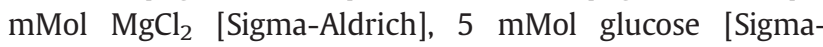
Aldrich]), pelleted again (380 g, 20 minutes) and re-suspended in $500 \mu \mathrm{L}$ Tyrode's-HEPES buffer. Platelets were diluted to a final concentration of $5 \times 10^{4}$ platelet $/ \mu$ L with Tyrode's-HEPES buffer. To stain RNA, $10 \mu \mathrm{L}$ of SYTO $13(1 \mu \mathrm{M}$, final concentration $12.5 \mathrm{nM}$; Thermo Fisher, Waltham, Massachusetts, United States) were added to a suspension of $100 \mu \mathrm{LWP}$ diluted in 690 $\mu \mathrm{L}$ phosphate-buffered saline (PBS; $\mathrm{pH} 7.4 ; 137 \mathrm{mMol} \mathrm{NaCl}, 2.7$ $\mathrm{mMol} \mathrm{KCl}, 10 \mathrm{mMol} \mathrm{Na}_{2} \mathrm{HPO}_{4}$ [Sigma-Aldrich], $1.8 \mathrm{mMol}$ $\mathrm{KH}_{2} \mathrm{PO}_{4}$ [EMD Millipore, Burlington, Massachusetts, United States]). For TO staining, $70 \mu \mathrm{L}$ of Retic-Count (Becton Dickinson, Heidelberg, Germany) was added to a suspension of 100 $\mu \mathrm{LWP}$ diluted in $630 \mu \mathrm{L}$ PBS. As negative controls, $100 \mu \mathrm{LWP}$ were diluted in $700 \mu \mathrm{L}$ PBS. After respective periods of incubation at room temperature in the dark, median fluorescence intensity (MFI, arbitrary units) of all platelets was measured using a SONY SH800Z cell sorter (SONY, Tokyo, Japan).

Furthermore, modifiability of assays is a desirable criterion for laboratory experiments. Hence, we compared staining of WP (a), fixed WP (b), PRP (c) and whole blood (d) to confirm adaptability of SYTO 13 staining of platelets:

(a) WP were prepared and stained for 90 minutes as aforementioned.

(b) After fixation of WP with $1 \%$ formaldehyde (Polysciences, Hirschberg, Germany) for 10 minutes, $100 \mu \mathrm{L}$ fixed WPs were stained with $10 \mu \mathrm{L}$ SYTO $13(1 \mu \mathrm{M})$ in $690 \mu \mathrm{L}$ PBS for 90 minutes.

(c) $50 \mu \mathrm{L}$ PRP were stained with $10 \mu \mathrm{L}$ SYTO $13(1 \mu \mathrm{M})$ in 690 $\mu \mathrm{L}$ PBS for 90 minutes.

(d) $5 \mu \mathrm{L}$ whole blood were double-stained with $5 \mu \mathrm{L}$ CD41 allophycocyanin (APC) (Becton Dickinson) as a plateletspecific marker and $10 \mu \mathrm{L}$ SYTO $13(1 \mu \mathrm{M})$ in $690 \mu \mathrm{L}$ PBS for 90 minutes.

All steps were performed at room temperature unless indicated otherwise. Fluorescence was quantified in the FL-1 fluorescein isothiocyanate channel $(500-550 \mathrm{~nm})$ after platelet gating by forward scatter/backward scatter characteristics and doublet exclusion. For whole blood staining, platelets were additionally identified by gating for CD41positivity in the FL-4 APC (650-680 nm) channel. Fluorescence intensities of unstained platelets remained stable over at least 24 hours. The sorter was calibrated with automatic setup beads (SONY) every day before use.

\section{Platelet RNA Stability at Different Incubation Temperatures}

RNA of unstained WP from three healthy donors $\left(19.5 \times 10^{6}\right.$, $21 \times 10^{6}, 23 \times 10^{6}$ platelets) was isolated immediately after WP preparation as well as after 5 hours of storage at $22^{\circ} \mathrm{C}$ or $37^{\circ} \mathrm{C}$, respectively. $500 \mu \mathrm{LWP}$ were diluted $1: 4$ with TRIzol LS reagent (Thermo Fisher). $200 \mu \mathrm{L}$ chloroform (Carl Roth, Karlsruhe, Germany) per $750 \mu$ L TRIzol LS were added. After 3 minutes of incubation, samples were centrifuged for 15 minutes at $12,000 \times g$ at $4^{\circ} \mathrm{C}$. The aqueous phase was manually separated. Thereafter, RNA isolation from the aqueous phase was accomplished by use of a miRNeasy Micro Kit (Qiagen, Venlo, Netherlands) per protocol of the manufacturer. The extracted RNA amount was determined by means of a RNA 6000 Pico Assay on a 2100 Bioanalyzer (Agilent, Santa Clara, California, United States).

\section{Correlation of TO/SYTO 13 Staining to IPF}

Blood samples obtained from 21 patients after transcatheter aortic valve implantation (TAVI) were analysed. Their 
median age was 83 years (interquartile range [IQR]: 80-86). Eighteen patients received Edwards-Sapien 3 aortic valves and 3 patients received Evolute R aortic valves, respectively. Demographic and clinical data of these patients are provided in - Supplementary Table $\mathbf{S 1}$ (available in the online version). The study was approved by the ethics committee of the University of Freiburg (Germany) and all patients gave written informed consent before any study procedure. Blood was collected 1 day following the TAVI procedure and WP were stained with TO and SYTO 13 as described afore. Polymethine-based RP analysis as well as IPF were determined using the automated blood cell counter SYSMEX XE2100. Spearman's $\rho$ was used to assess correlations between IPF and MFI of TO- and SYTO 13-stained platelets.

\section{RNA Quantification according to SYTO 13 Staining Intensities}

For internal validation, the association of RNA content and SYTO 13 staining intensities was investigated. SYTO 13stained platelets obtained from a drug-free healthy subject were divided into quintiles in the FL-1 channel according to increasing fluorescence intensities. A total of $8 \times 10^{6}$ platelets were sorted from each gate and RNA was extracted as described before. Due to the high amount of fluid, the first centrifugation step in the Qiagen protocol was modified and a vacuum chamber was used instead for separation of RNA. The next steps were performed per protocol of the manufacturer. The extracted RNA amount was determined in duplicate as described before.

\section{RNA Quantification of RNAlow and RNArich Platelets}

As a second internal validation, the RNA amount of $8 \times 10^{6}$ platelets sorted from the outer quintiles of SYTO 13 fluorescence intensities (termed RNAlow and RNArich platelets) was quantified in WP from eight healthy donors. RNA was extracted and quantified as described before. Paired $t$-test (GraphPad Prism 7, San Diego, California, United States) was used to compare RNA amounts of RNAlow and RNArich platelets. All values are expressed as median with IQR unless otherwise indicated.

\section{Results}

\section{Staining of Washed Platelets}

SYTO 13 staining of platelets showed a continuous shift in the FL-1 channel with-compared with TO staining-a remarkable 10-fold increase in mean fluorescence intensity (-Fig. 1). Doublet gating was used to avoid staining events with false positive high fluorescence intensity (-Supplementary Fig. S1, available in the online version).

\section{Staining under Various Conditions}

Furthermore, staining of fixed WP, PRP and whole blood showed that SYTO 13 staining is feasible under various conditions. Each tested condition yields an increase of fluorescence in the FL-1 channel (- Fig. 2, left two columns). In sorting experiments, SYTO 13 staining of platelets enables adequate sorting as confirmed by re-analysis of flow cytometry plots for RNArich and RNAlow platelets in different FL1 gates ( - Fig. 2, right two columns). Platelet fixation resulted in loss of MFI as compared with the other conditions. Depending on the planned specific laboratory experiments, one might choose the most appropriate condition.

\section{Stability of Staining Intensity}

Having demonstrated that SYTO 13 staining, as compared with TO staining, is feasible after 90 minutes of staining, the stability of staining intensity over prolonged incubation times was investigated. After staining for 90 minutes, fluorescence intensity remained stable for at least 5 hours ( - Fig. 3A). RNA amount remained stable up to at least 5 hours of incubation at room temperature $\left(22^{\circ} \mathrm{C}\right)$, while a substantial amount of RNA was degraded after 5 hours of incubation at $37^{\circ} \mathrm{C}$ (-Fig. 3B).

\section{Correlation of TO and SYTO 13 Staining with IPF}

MFI of SYTO 13-stained platelets correlated well with the established IPF measurement by SYSMEX analysed $30 \mathrm{~min}-$ utes $(r=0.665, p=0.001)$ as well as 90 minutes $(r=0.668$, $p<0.001)$ after staining, whereas a moderate correlation with MFI of TO-stained platelets was obtained solely after 30 minutes $(r=0.478, p=0.029 ;$ - Fig. 4).
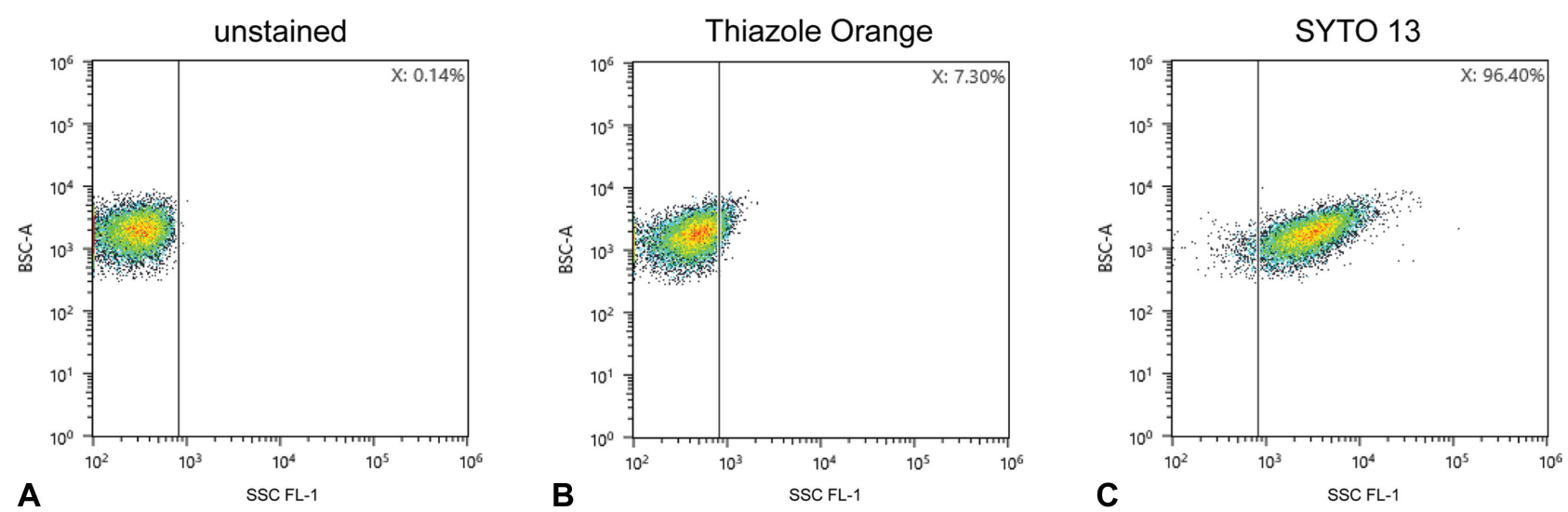

Fig. 1 Flow cytometry plots after an incubation time of 90 minutes of (A) unstained, (B) TO-stained and (C) SYTO 13-stained unfixed washed platelets from a patient after TAVI. Abbreviations: BSC, backward scatter; SSC, side scatter; FL-1, 500-550 nm; TAVI, transcatheter aortic valve implantation; TO, thiazole orange. 
unstained

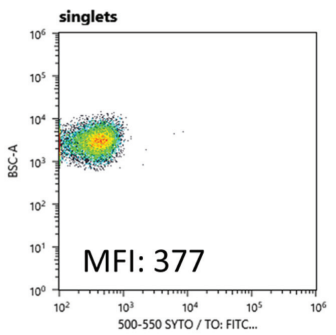

washed

platelets

\section{fixed washed} platelets

platelet rich plasma
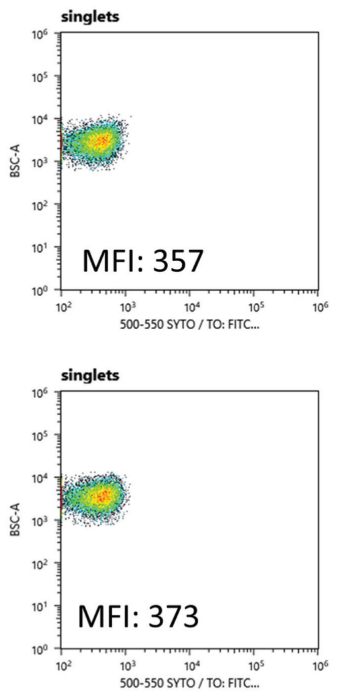

whole blood, CD41-positive platelets
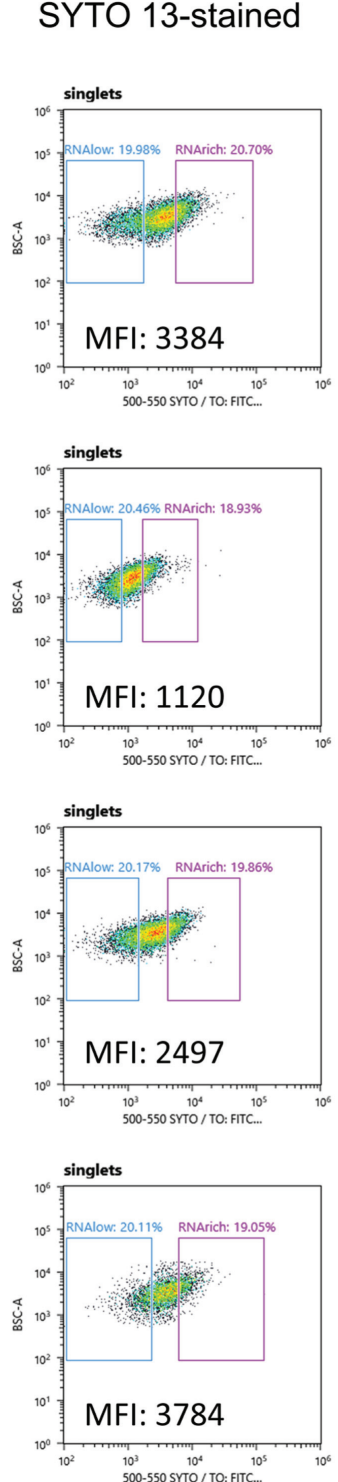

reanalysis:

RNAlow platelets
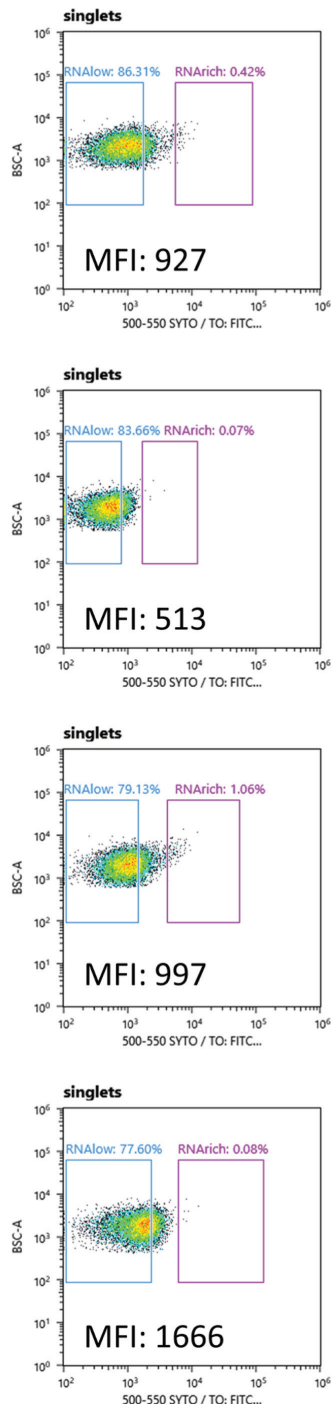

reanalysis:

RNArich platelets
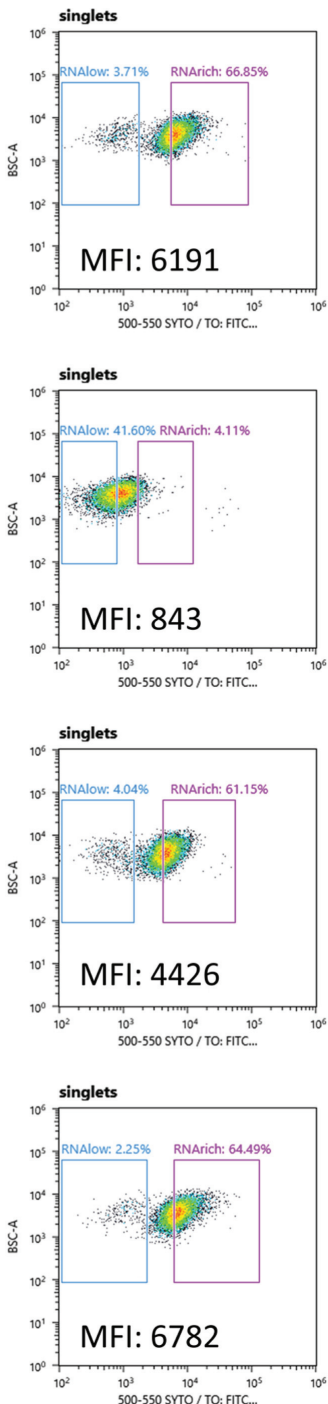

Fig. 2 Staining of washed platelets, fixed washed platelets, platelet-rich plasma and whole blood with SYTO 13 for 90 minutes. Platelets were sorted according to the RNAlow and RNArich gates and re-analysed. MFI, median fluorescence intensity (arbitrary units).
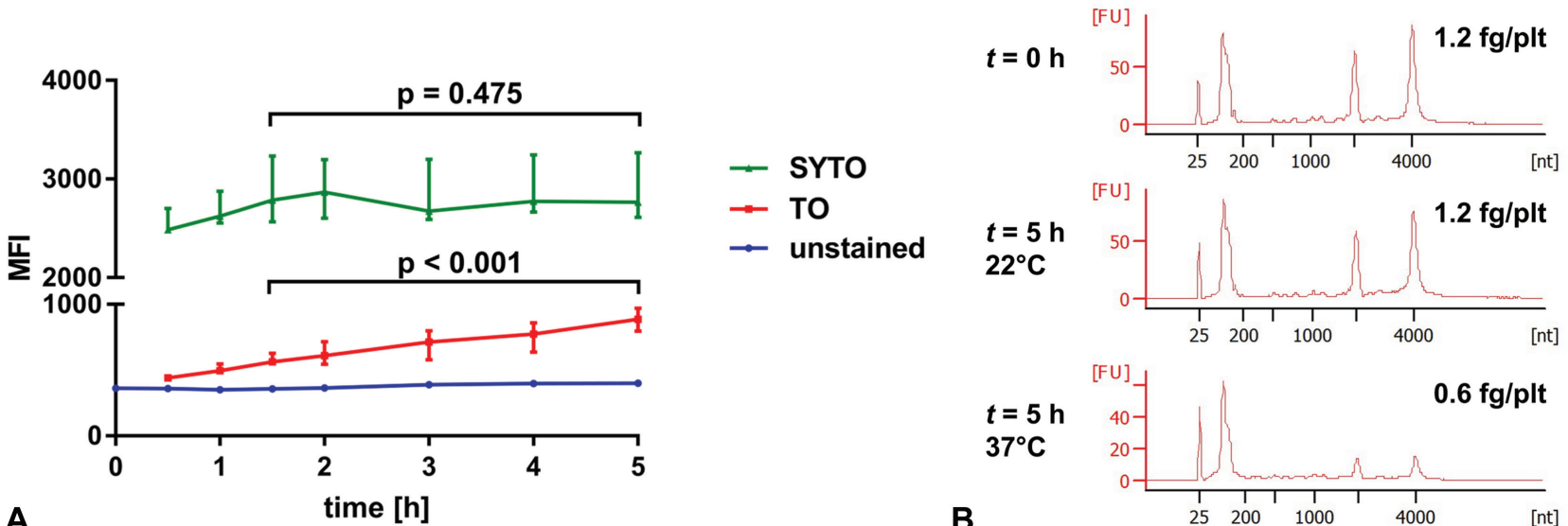

$t=5 \mathrm{~h}$
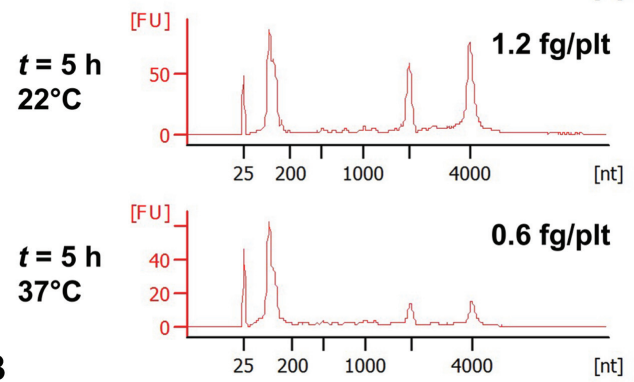

Fig. 3 (A) Stability of SYTO 13 and thiazole orange (TO) staining over time from 3 healthy subjects (median and range; $h=$ hours). (B) Representative example of a Bioanalyzer electropherogram of washed platelet ribonucleic acid (RNA) from a healthy donor immediately after preparation of washed platelets and at 5 hours of storage at $22^{\circ} \mathrm{C}$ and $37^{\circ} \mathrm{C}$, respectively. Abbreviation: MFI, median fluorescence intensity (arbitrary units). 

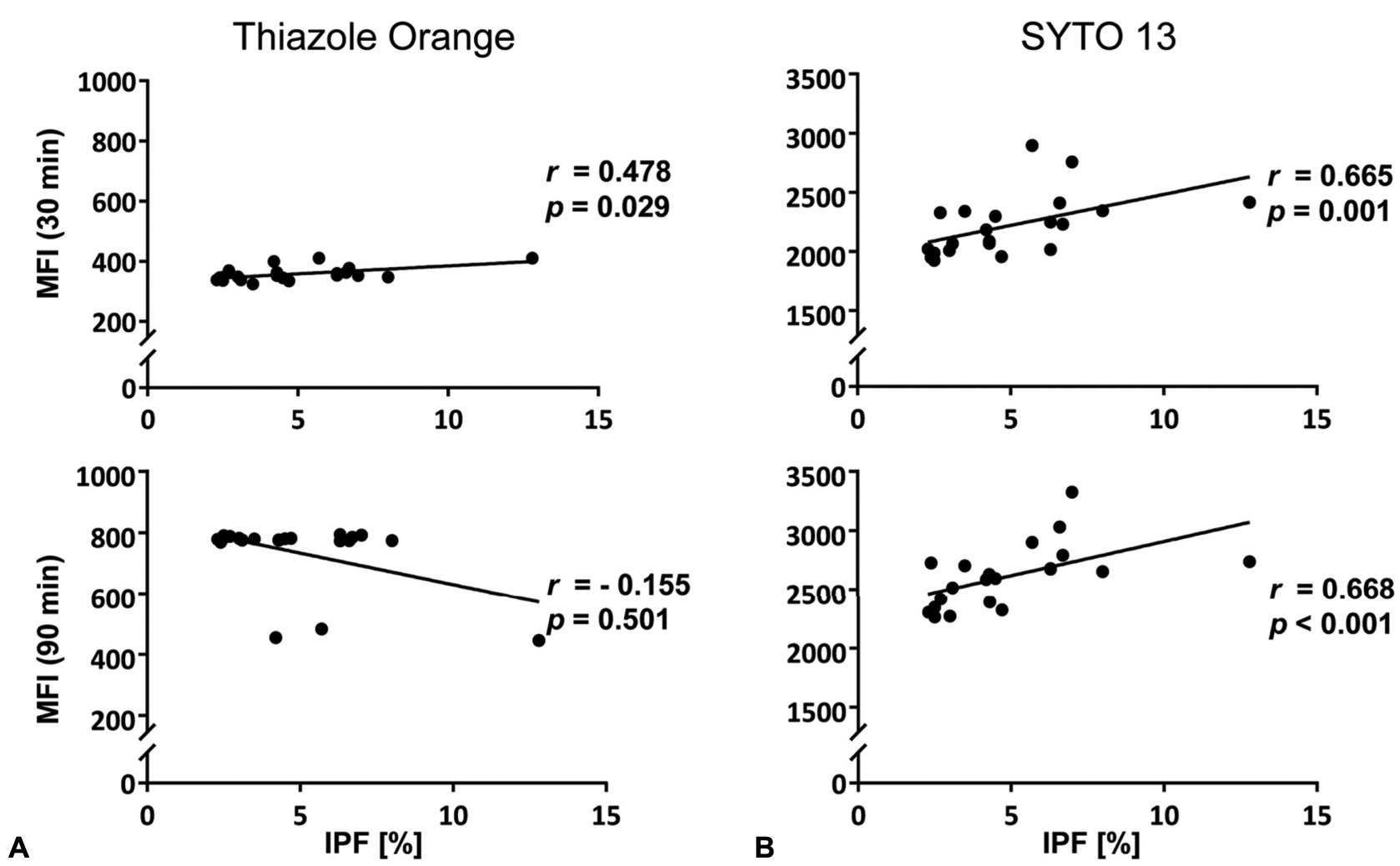

Fig. 4 Correlation of immature platelet fraction (IPF) and MFI of unfixed washed platelets after 30 minutes (upper panels) and 90 minutes (lower panels) of staining with (A) thiazole orange and (B) SYTO $13 ; n=21$ patients on day 1 after transcatheter aortic valve implantation. Abbreviation: MFI, median fluorescence intensity (arbitrary units).

\section{RNA Quantification according to SYTO 13 Staining Intensities}

RNA quantification of sorted platelets according to the quintiles of MFI showed a strong association of SYTO 13 staining intensities and extracted RNA amount (-Fig. 5). - Fig. 5B shows Bioanalyzer electropherograms of sorted platelets from each quintile indicating an increase in the amount of total RNA with higher fluorescence intensity. $18 \mathrm{~S}$ and $28 \mathrm{~S}$ ribosomal peaks can only be observed in the electropherogram of the quintile with highest staining intensity which is indicative for the high RNA amount in this quintile.

RNA Quantification of RNAlow and RNArich Platelets RNA quantification resulted in a RNA amount of 0.35 (0.230.52 ) fg per platelet in the $20 \%$ of platelets with lowest staining intensity (RNAlow platelets) and 0.88 (0.78-1.14) fg per platelet in the $20 \%$ of platelets with highest staining intensity (RNArich platelets; $p=0.016$ ).

\section{Discussion}

Our approach was based on certain features: (1) the staining dye should be specific for a single component of the platelet, (2) the staining procedure should yield a clear increase in fluorescence intensity, (3) the staining conditions should enable additional staining of extra- and intracellular components with other dyes or antibodies, (4) advanced processing of platelets by cell sorting for further analysis should be feasible and (5) the staining should be comparable to the well stan- dardized and clinically established method, the SYSMEX polymethine dye-based assay. We selected a nucleotide staining dye since this seems to fulfil all requirements outlined above.

RNA extraction and quantification of platelets divided into quintiles by fluorescence intensity after SYTO 13 staining showed a clear association of RNA amount and fluorescence intensity. Distinct ribosomal peaks could only be determined in the electropherogram of the quintile with highest fluorescence intensity indicating that RPs are present in this quintile. Hence, the quintile with highest fluorescence intensity may be adopted as a gate for dedicated sorting of RP. RNA extraction and quantification of sorted RNAlow and RNArich platelets confirm highly different amounts of RNA in the respective populations. Therefore, our staining and gating method indeed identifies different platelet populations having low and high RNA levels correctly. In contrast to TO staining, the stability of SYTO 13 MFI facilitates analysis of stained platelets in extended experimental settings, for example, during cell sorting with low event rates.

Despite the strong association of SYTO 13 fluorescence intensities and extracted RNA amount, we cannot exclude potential unspecific labelling of other cell components, for example, dense granules or mitochondria. From the current data, similar to other methods with continuous staining distribution, the true rate of newly released platelets per day remains undetermined. Detailed in vivo experiments, for example, radiolabelling, would be necessary to define specific cut-off values for platelet turnover. In summary, our results indicate that SYTO 13 seems to be an attractive alternative staining dye for experiments on RNA-rich platelets and thereby also for RP. 
A
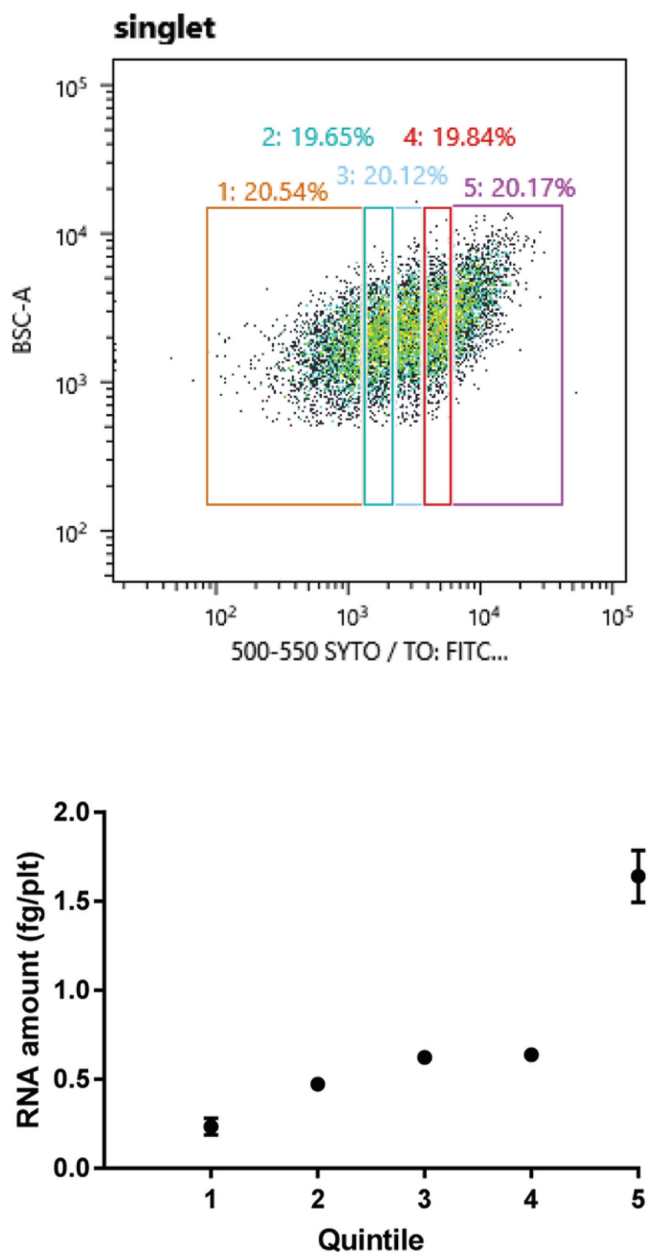
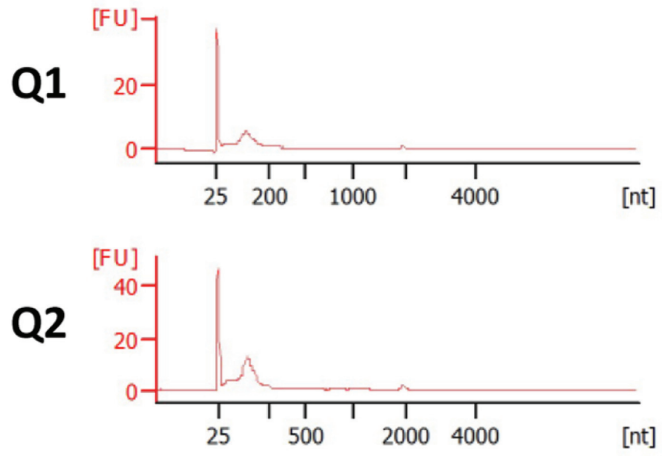

Q3

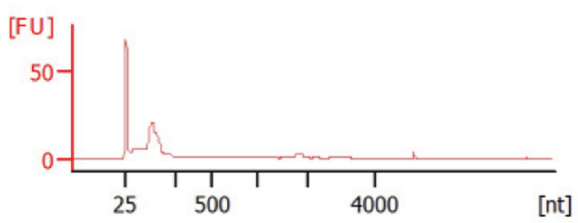

Q4

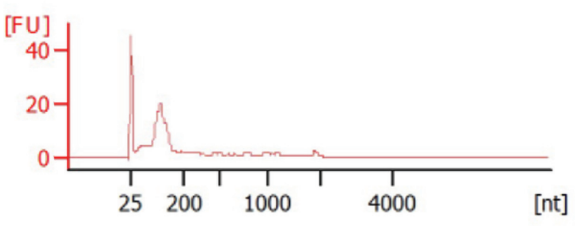

Q5

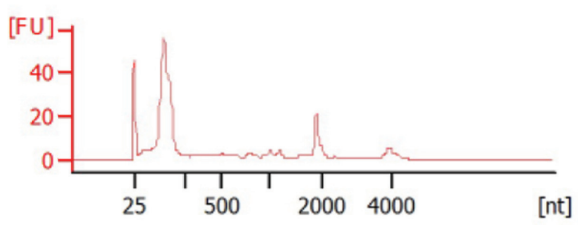

B

Fig. 5 (A) Gating strategy for sorting unfixed washed platelets in the FL-1 channel according to quintiles ranging from low to high fluorescence intensities after SYTO 13 staining (incubation time: 90 minutes). (B) Bioanalyzer electropherograms of extracted platelet ribonucleic acid (RNA) from each quintile. (C) RNA amount (fg per platelet) of sorted platelets from each quintile; platelets from a drug-free healthy subject measured in duplicate.

\section{What is known about this topic?}

- A higher immature platelet fraction (IPF) is linked to higher platelet reactivity.

- Reticulated platelets are associated with increased cardiovascular events and mortality.

- There is no generally accepted standardised protocol for the determination of reticulated platelets using thiazole orange (TO) despite this is the so far most frequently used non-automated laboratory method.

\section{What does this paper add?}

- An alternative and highly stable staining method for reticulated platelets using SYTO 13 is described.

- Fluorescence intensities of SYTO 13-stained platelets are associated with the clinically so far best standardised laboratory parameter IPF.

- In contrast to TO staining, SYTO 13 staining facilitates analysis of platelets in extended experimental settings.
Note

Part of the data was presented as a poster at the Congress of the European Society of Cardiology 2017 in Barcelona (Spain).

\section{Funding}

This study was supported by the PharmCompNet BadenWürttemberg: Kompetenznetzwerk Pharmakologie Baden-Württemberg - Wirkstoffnetzwerke als Grundlagen der individualisierten Arzneistofftherapie and the University Heart Center Freiburg - Bad Krozingen.

\section{Conflict of Interest}

Miss Hille reports grants from PharmCompNet BadenWürttemberg: Kompetenznetzwerk Pharmakologie BadenWürttemberg, grants from University Heart Center FreiburgBad Krozingen, during the conduct of the study.

Dr. Trenk reports grants fromPharmCompNet BadenWürttemberg:Kompetenznetzwerk Pharmakologie BadenWürttemberg during the conduct of the study; personal fees from Amgen, AstraZeneca, Bayer, Berlin Chemie, Boehringer 
Ingelheim, Bristol-Myers Squibb, Daiichi Sankyo, Pfizer and Sanofi, outside the submitted work.

Dr. Cederqvist, Dr. Hromek, Dr. Stratz and Dr. Nührenberg report no conflicts of interest.

\section{References}

1 Hoffmann JJ. Reticulated platelets: analytical aspects and clinical utility. Clin Chem Lab Med 2014;52(08):1107-1117

2 D'Souza C, Briggs C, Machin SJ. Platelets: the few, the young, and the active. Clin Lab Med 2015;35(01):123-131

3 Perl L, Lerman-Shivek H, Rechavia E, et al. Response to prasugrel and levels of circulating reticulated platelets in patients with STsegment elevation myocardial infarction. J Am Coll Cardiol 2014; 63(06):513-517

4 Angénieux C, Maître B, Eckly A, Lanza F, Gachet C, de la Salle H. Time-dependent decay of mRNA and ribosomal RNA during platelet aging and its correlation with translation activity. PLoS One 2016;11(01):e0148064

5 Stratz C, Bömicke T, Younas I, et al. Comparison of immature platelet count to established predictors of platelet reactivity during thienopyridine therapy. J Am Coll Cardiol 2016;68(03):286-293

6 Armstrong PC, Hoefer T, Knowles RB, et al. Newly formed reticulated platelets undermine pharmacokinetically short-lived antiplatelet therapies. Arterioscler Thromb Vasc Biol 2017;37(05):949-956

7 Lakkis N, Dokainish H, Abuzahra M, et al. Reticulated platelets in acute coronary syndrome: a marker of platelet activity. J Am Coll Cardiol 2004;44(10):2091-2093

8 Grove EL, Hvas AM, Kristensen SD. Immature platelets in patients with acute coronary syndromes. Thromb Haemost 2009;101(01): 151-156
9 Cesari F, Marcucci R, Gori AM, et al. Reticulated platelets predict cardiovascular death in acute coronary syndrome patients. Insights from the AMI-Florence 2 Study. Thromb Haemost 2013;109(05):846-853

10 Ibrahim H, Schutt RC, Hannawi B, DeLao T, Barker CM, Kleiman NS. Association of immature platelets with adverse cardiovascular outcomes. J Am Coll Cardiol 2014;64(20):2122-2129

11 Ingram $\mathrm{M}$, Coopersmith $\mathrm{A}$. Reticulated platelets following acute blood loss. Br J Haematol 1969;17(03):225-229

12 Kienast J, Schmitz G. Flow cytometric analysis of thiazole orange uptake by platelets: a diagnostic aid in the evaluation of thrombocytopenic disorders. Blood 1990;75(01):116-121

13 Robinson MS, Mackie IJ, Khair K, et al. Flow cytometric analysis of reticulated platelets: evidence for a large proportion of nonspecific labelling of dense granules by fluorescent dyes. $\mathrm{Br} \mathrm{J}$ Haematol 1998;100(02):351-357

14 Ault KA, Rinder HM, Mitchell J, Carmody MB, Vary CP, Hillman RS. The significance of platelets with increased RNA content (reticulated platelets). A measure of the rate of thrombopoiesis. Am J Clin Pathol 1992;98(06):637-646

15 Dale GL, Friese P, Hynes LA, Burstein SA. Demonstration that thiazole-orange-positive platelets in the dog are less than 24 hours old. Blood 1995;85(07):1822-1825

16 Guthikonda S, Alviar CL, Vaduganathan M, et al. Role of reticulated platelets and platelet size heterogeneity on platelet activity after dual antiplatelet therapy with aspirin and clopidogrel in patients with stable coronary artery disease. J Am Coll Cardiol 2008;52 (09):743-749

17 Bernlochner I, Goedel A, Plischke C, et al. Impact of immature platelets on platelet response to ticagrelor and prasugrel in patients with acute coronary syndrome. Eur Heart J 2015;36 (45):3202-3210 